\title{
Adaptive Countering Technique for Angle Deception Based on Dual Polarization Radar Seeker
}

\author{
Qiang Zhang and Weijun Pan \\ College of Air Traffic Management, Civil Aviation Flight University of China, Guanghan 618307, China \\ Correspondence should be addressed to Qiang Zhang; zhangqiang_ioe@126.com
}

Received 27 December 2016; Revised 1 April 2017; Accepted 2 May 2017; Published 22 May 2017

Academic Editor: Pierfrancesco Lombardo

Copyright (C) 2017 Qiang Zhang and Weijun Pan. This is an open access article distributed under the Creative Commons Attribution License, which permits unrestricted use, distribution, and reproduction in any medium, provided the original work is properly cited.

\begin{abstract}
Angle deception jamming makes the monopulse radar seeker track to itself but not the real target, which is catastrophic for the guidance radar. In this paper, an adaptive technique based on dual polarization radar is presented to counter it. How angle deception jamming acts on the monopulse tracking radar is first investigated. An angle estimation technique of the real target is then derived from the conventional monopulse method, although it is being interfered with by angle deception jamming. Meanwhile, the polarization ratio characteristic of the angle deception jamming could be adaptively estimated in current practical scene. Furthermore, the similar characteristic of Jones vectors is defined as the rule to judge whether the target is being interfered with by jamming. It can make the radar seeker select different techniques for angle estimation adaptively. Finally, two major factors of angle estimation accuracy are analyzed by simulation and the effectiveness of the proposed technique is proved through experiments.
\end{abstract}

\section{Introduction}

Angle deception jamming is a significant electromagnetic interference for monopulse radar seekers, which is in the same range unit and beam unit with the target, and making the seekers track to themselves but not the real target. In [1-4], the direction of arrival (DOA) estimation of two unresolved targets was developed, but the estimation techniques were for the same type targets and needed radar cross sections (RCSs) as prior information. However, including chaff clouds, active jamming, corner reflectors, arbitrary combinations of them, and so forth, angle deception jamming is quite different from the real targets. What is more, the RCSs of jamming and targets could not be obtained priorly. A main-lobe jamming suppression technique based on the spatial polarization characteristics (SPCs) of the antenna of a single polarization radar seeker was proposed [5-7]. It can suppress the echo signal from main-lobe jamming and obtain the DOA of the target by orthogonal polarization decomposition, polarization estimation, and spatial virtual polarization filtering. Whereas the technique needs to know the direction of jamming and the SPCs are just present in offset reflector antennas. In [8], a detection technique for centroid chaff jamming was developed, while it needed information from not only the monopulse radar but also GPS (Global Position System) or INS (Inertial Navigation System). As mentioned above, information acquired by single polarization radar seekers is not sufficient, so multipolarization radar seekers with the ability of achieving more polarization information would be widely chosen to counter interference, improve recognition rate of targets, and so forth [9]. According to public reporting, there are a radar seeker at $94 \mathrm{GHz}$ carried by a brimstone missile [10] and a multirole modular seeker (MRMS) [11] on active service. They are all in possession of dual polarization system and developed by MBDA missile systems. Dual polarization radar seekers usually possess two orthogonal polarization channels all with sum, azimuth difference, and elevation difference, which differ greatly from the conventional monopulse radar seekers with single polarization. A scheme of adaptive polarization filtering based on dual polarization radar seeker was put forward in [12]. The polarization characteristics could be dynamically acquired by tracking jamming and the target with Kalman estimator, and then the polarization filter was utilized to suppress angle deception jamming. Yet the angle estimation technique for the real target is not provided. 
In [13-16], oblique projection technology was introduced into the polarization domain. It employed disjoint polarization subspaces to construct polarization oblique projection operator, which could remain undistorted target signal and absolutely suppress jamming signal, whereas the polarization oblique projection operator based on dual polarization radar is very sensitive for estimation error of the polarization characteristic.

This paper puts forward an adaptive countering technique for angle deception jamming based on dual polarization radar seeker. It can be utilized to acquire the actual angle information of the real target under angle deception jamming without any prior knowledge. The paper provides a study of the countering technique just in azimuth, since it is also valid in elevation. The remainder of this paper is organized as follows. In Section 2, the theory of angle deception jamming for monopulse tracking radars is revealed. Section 3 addresses the angle estimation technique for the real target being interfered with by angle deception jamming based on dual polarization radar. Then an adaptive countering technique by establishing a judgmental rule is formulated in Section 4. Angle estimation error analyses and experimental results are demonstrated in Sections 5 and 6. Finally, Section 7 concludes the paper.

\section{Theory of Angle Deception}

For monopulse radars with sum and difference patterns of amplitude, their measurements of the target angle depend on the ratio of difference signal to sum signal. Being smaller than the width of the sum beam, the angular deviation of the target from the radar boresight obeys approximately linear relationship to the ratio of difference signal to sum signal. Thus the principle of monopulse angle measurement is defined as

$$
k \theta=\frac{\Delta}{\Sigma},
$$

where $\Delta$ represents the difference signal, $\Sigma$ represents the sum signal, $\theta$ is the angular deviation of the target from the radar boresight, $k$ is the difference slope constant closely related to the radar system $[17,18]$.

When the target is being interfered with by angle deception jamming, such as corner reflectors, chaff clouds, active jamming, and arbitrary combinations of them, the difference signal and the sum signal received by the radar are all composed of echoes from both the target and jamming. As a result, the sum signal can be defined as

$$
\Sigma_{(T+I)}=\Sigma_{T}+\Sigma_{I}
$$

Something to note in the whole paper is that subscript $T$ denotes the target, $I$ denotes jamming, and $(T+I)$ denotes the target and jamming existing simultaneously.

Combining (1) with (2) gives us the difference signal expression as

$$
\Delta_{(T+I)}=\Delta_{T}+\Delta_{I}=k\left(\alpha \Sigma_{T}+\beta \Sigma_{I}\right),
$$

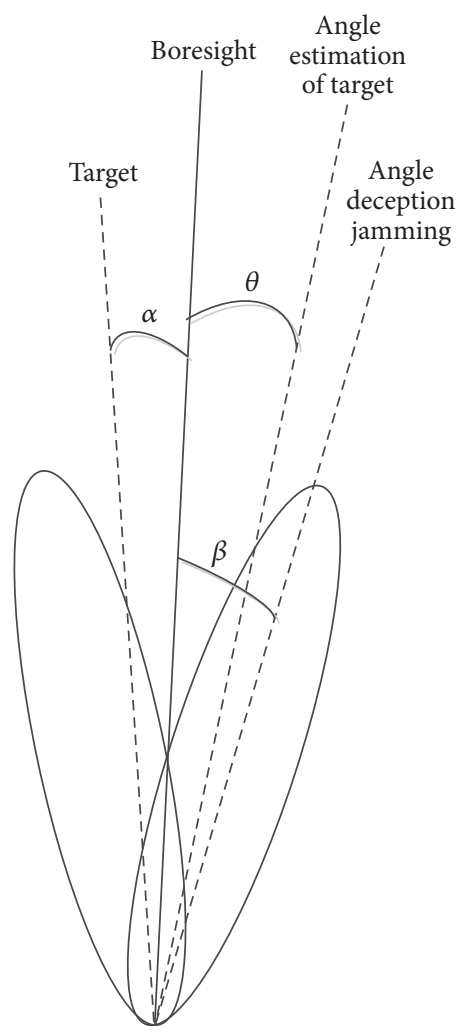

Figure 1: Schematic diagram of angle deception.

where $\alpha$ and $\beta$ are respective angular deviations of the target and jamming from the radar boresight.

The complex ratio of jamming signal to target signal is expressible as

$$
\frac{\Sigma_{I}}{\Sigma_{T}}=r e^{j \emptyset},
$$

where $r$ is the amplitude ratio of them and $\emptyset$ is the phase difference between them. Notably, the value of $r$ is great because jamming usually has a much larger RCS than the target.

Substitutions of (2), (3), and (4) into (1) yield (5). When RCS of angle deception jamming is much larger than that of the target, (5) can be simplified as (6). The result of angle measurement is much closer to the jamming but not the target. Then, the monopulse radar will track to the angle deception jamming.

$$
\begin{aligned}
\frac{\Delta_{(T+I)}}{\Sigma_{(T+I)}} & =k \frac{\alpha \Sigma_{T}+\beta \Sigma_{I}}{\Sigma_{T}+\Sigma_{I}}=k \frac{\alpha+\beta r e^{j \emptyset}}{1+r e^{j \emptyset}} \\
& =\frac{k \alpha}{1+r e^{j \emptyset}}+\frac{k \beta r e^{j \emptyset}}{1+r e^{j \emptyset}} \\
\frac{\Delta_{(T+I)}}{\Sigma_{(T+I)}} & \approx k \beta \quad(\text { when } r \gg 1) .
\end{aligned}
$$

The schematic diagram of angle deception jamming is shown in Figure 1. The angle value of the target acquired by 
the monopulse radar is the DOA of the signal contributed by the target and jamming together. The real target and the angle deception jamming are, respectively, with the angle of $\alpha$ and $\beta$ from the boresight. $\theta$ is the angle estimation value of the target under jamming, which is much closer to the angle deception jamming. In other words, if the value of $\alpha$ can be calculated by some means, which means the angle deception jamming has no influence on the monopulse tracking radar.

\section{Countering Technique for Angle Deception Jamming}

The dual polarization radar is a vertical polarization transmitting but both vertical and horizontal polarization receiving system. Therefore, its sum and difference signals from vertical and horizontal polarization channels result, respectively. According to (2) and (3) in Section 2, sum and difference signals of vertical polarization channel can be denoted as (7) and (8), and identically those of horizontal polarization channel can be denoted as (9) and (10).

$$
\begin{aligned}
& \Sigma_{(T+I) v v}=\Sigma_{T v v}+\Sigma_{I v v} \\
& \Delta_{(T+I) v v}=\Delta_{T v v}+\Delta_{I v v}=k\left(\alpha \Sigma_{T v v}+\beta \Sigma_{I v v}\right) \\
& \Sigma_{(T+I) h v}=\Sigma_{T h v}+\Sigma_{I h v} \\
& \Delta_{(T+I) h v}=\Delta_{T h v}+\Delta_{I h v}=k\left(\alpha \Sigma_{T h v}+\beta \Sigma_{I h v}\right) .
\end{aligned}
$$

Here, subscript $v v$ represents vertical polarization transmitting and vertical polarization receiving and $h v$ represents vertical polarization transmitting but horizontal polarization receiving. For instance, $\Sigma_{(T+I) h v}$ represents the sum signal obtained by the horizontal polarization channel when the target and jamming are simultaneously irradiated by vertical polarization wave in the same beam.

For monopulse tracking systems of dual polarization radars, they usually possess four working stages of search, detection, recognition, and tracking. It is worth noting that this paper just focuses on studying how not to be attracted to the angle deception jamming from the real target in the tracking stage. As the inputs of the tracking stage, the parameters of the real target are acquired through the previous stages.

In addition, the polarization ratio is a characteristic to describe the converting polarization ability of radar targets, which is the main polarization characteristic obtained by the dual polarization radar. As a matter of fact, the polarization ratio of the jamming can be calculated by

$$
p_{I}=\frac{\Sigma_{I v v}}{\Sigma_{I h v}}=\frac{\sum_{(T+I) v v}-\Sigma_{T v v}}{\sum_{(T+I) h v}-\Sigma_{T h v}},
$$

where $p_{I}$ denotes the polarization ratio of the jamming, $\Sigma_{T v v}$ and $\Sigma_{T h v}$ can be obtained after the target is recognized, and then $\Sigma_{(T+I) v v}$ and $\Sigma_{(T+I) h v}$ can be obtained in the tracking stage when the target is being interfered with by jamming. Thus, the polarization ratio characteristic of the jamming can be adaptively estimated in current practical scene. Especially, without any prior knowledge, the polarization ratio characteristic obtained by the method would be more reliable and universal.

Then, solving (7), (8), (9), (10), and (11), $\alpha$ is calculated by (12) (the derivation process is accorded in Appendix).

$$
\alpha=\frac{1}{k} \frac{\Delta_{(T+I) v v}-p_{I} \Delta_{(T+I) h v}}{\sum_{(T+I) v v}-p_{I} \sum_{(T+I) h v}} .
$$

Here $\alpha$ is the actual angle of the real target under angle deception jamming, $p_{I}$ results by (11), and the other four parameters are provided by two channels of the dual polarization radar. Consequently, the proposed technique can counter angle deception jamming and obtain the actual angle of the real target.

\section{Adaptive Processing Technique}

While tracking a target, the dual polarization radar seeker does not know whether the target is being interfered with by angle deception jamming and especially when to apply the proposed countering technique. In this paper, it is accomplished by constructing a judgmental rule.

Equations (13) are the $m$ th and $(m+1)$ th estimations of the complex Jones vectors [19] of the target signals, which are replaced by their real signals obtained by the dual polarization radar.

$$
\begin{aligned}
S(m) & =\left[\begin{array}{ll}
\Sigma_{h v}(m) & \Sigma_{v v}(m)
\end{array}\right] \\
S(m+1) & =\left[\begin{array}{lll}
\Sigma_{h v}(m+1) & \Sigma_{v v}(m+1)
\end{array}\right] .
\end{aligned}
$$

Angle cosine coefficient is introduced to denote the similar characteristic of the two Jones vectors. It can be accorded by

$$
\begin{aligned}
& \sigma_{p}=\cos \varphi=\frac{\left|S(m) S(m+1)^{T}\right|}{\|S(m)\|\|S(m+1)\|} \\
& =\frac{\left|\Sigma_{h v}(m) \Sigma_{h v}(m+1)+\Sigma_{v v}(m) \Sigma_{v v}(m+1)\right|}{\sqrt{\left(\left|\Sigma_{h v}(m)\right|^{2}+\left|\Sigma_{v v}(m)\right|^{2}\right)\left(\left|\Sigma_{h v}(m+1)\right|^{2}+\left|\Sigma_{v v}(m+1)\right|^{2}\right)}},
\end{aligned}
$$

where $\varphi$ is the angle between the two vectors of $S(m)$ and $S(m+1), \sigma_{p}$ is the cosine value of $\varphi$, superscript $T$ represents the transpose of a vector, $|\cdot|$ represents the absolute value, and $\|\cdot\|$ represents the Frobenius norm [20]. Evidently, $\sigma_{p} \leq 1$ is always correct. And the closer the value is to 1 , the less the difference between the two Jones vectors is. If it is not close to 1 , that means the target is being interfered with by jamming; otherwise it means not being interfered with by jamming. Hence, it is applicable to judge whether the target is being interfered with by angle deception jamming.

The adaptive processing technique for countering angle deception is expressible as

$$
\alpha= \begin{cases}\frac{1}{k} \frac{\Delta_{(T+I) v v}-p_{I} \Delta_{(T+I) h v}}{\sum_{(T+I) v v}-p_{I} \Sigma_{(T+I) h v}} & \sigma_{p} \leq T_{h} \\ \frac{\Delta_{(T+I) v v}}{k \sum_{(T+I) v v}} & \sigma_{p}>T_{h}\end{cases}
$$

where $\sigma_{p} \leq T_{h}$ means that the target is being interfered with by jamming, $\sigma_{p}>T_{h}$ means that the target is not being 
interfered with by jamming, and $T_{h}$ is usually determined as a value of 0.8 from experiences.

In practical application, the procedure of adaptive processing is descriptive in Figure 2. After the radar system recognizes the target, it will work in the tracking stage with the angle estimation of the conventional monopulse method. Meanwhile the angle cosine coefficient between $S(m)$ and $S(m+1)$ is consecutively calculated. If $\sigma_{p} \leq T_{h}$, the proposed countering technique will be applied to estimate the angle of the target. But the last measured value of $S(m)$ before the target is interfered with by jamming would be maintained, and it will be utilized to calculate the angle cosine coefficient with the present Jones vectors while the target is being interfered with by jamming. Then, if $\sigma_{p}>T_{h}$, the conventional monopulse method will be applied to estimate the angle of the target again. And, afterwards, the cycle repeats. Therefore, the angle estimation of the real target can be obtained through adaptively selecting the conventional monopulse method or the proposed countering technique.

\section{Angle Error Analyses after Countering Technique}

The polarization ratio is too significant a parameter for obtaining the real DOA of the target. Certainly, the estimation error of $p_{I}$ is a crucial factor for the angle estimation error after the countering technique. by

The estimation error of the target angle can be accorded

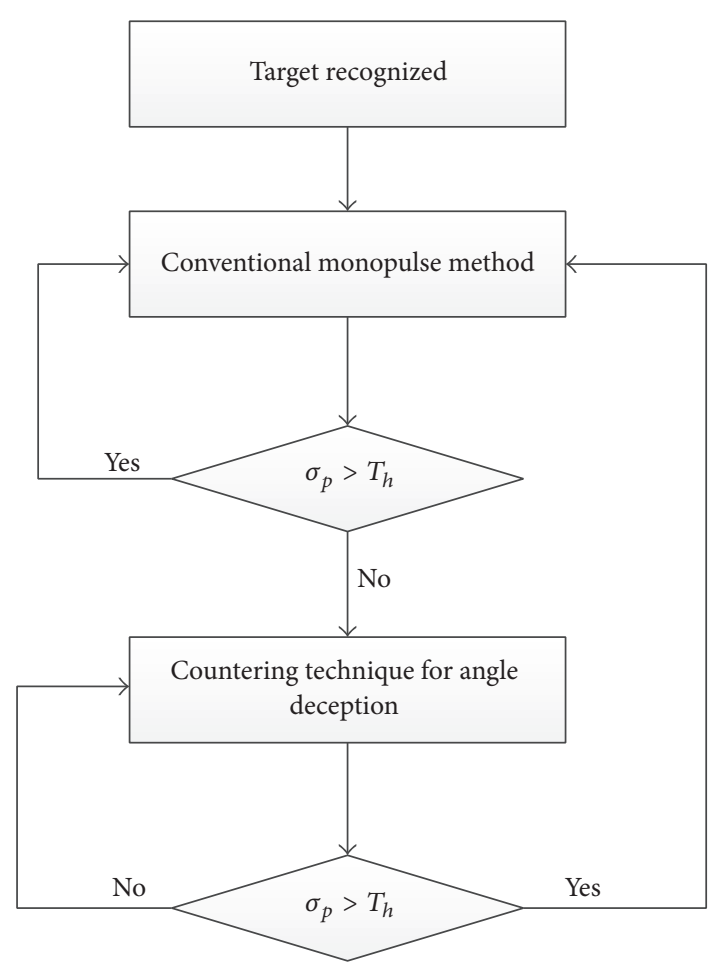

Figure 2: Procedure of adaptive processing technique.

$$
\begin{aligned}
|\Delta \alpha| & =|\widehat{\alpha}-\alpha|=\left|\frac{1}{k} \frac{\Delta_{(T+I) v v}-\widehat{p}_{I} \Delta_{(T+I) h v}}{\Sigma_{(T+I) v v}-\widehat{p_{I}} \Sigma_{(T+I) h v}}-\frac{1}{k} \frac{\Delta_{(T+I) v v}-p_{I} \Delta_{(T+I) h v}}{\sum_{(T+I) v v}-p_{I} \Sigma_{(T+I) h v}}\right| \\
& =\frac{1}{k}\left|\frac{\left(\Delta_{(T+I) v v} \Sigma_{(T+I) h v}-\Delta_{(T+I) h v} \Sigma_{(T+I) v v}\right)\left(\widehat{p}_{I}-p_{I}\right)}{\left(\sum_{(T+I) v v}-p_{I} \Sigma_{(T+I) h v}\right)^{2}+\left(\widehat{p_{I}}-p_{I}\right)\left(p_{I} \sum_{(T+I) h v}{ }^{2}-\Sigma_{(T+I) v v} \Sigma_{(T+I) h v}\right)}\right|,
\end{aligned}
$$

where $|\Delta \alpha|$ is the absolute value of the angle estimation error caused by the polarization ratio estimation error, $\widehat{\alpha}$ is the estimation of $\alpha$, and $\widehat{p_{I}}$ is the estimation of $p_{I}$.
Applying $\varepsilon$ to denote the polarization ratio estimation error of jamming; that is to say, $\varepsilon=\widehat{p_{I}}-p_{I}$, so (16) can be simplified as

$$
|\Delta \alpha|=\frac{1}{k}\left|\frac{\left(\Delta_{(T+I) v v} \Sigma_{(T+I) h v}-\Delta_{(T+I) h v} \Sigma_{(T+I) v v}\right) \varepsilon}{\left(\sum_{(T+I) v v}-p_{I} \Sigma_{(T+I) h v}\right)^{2}+\left(p_{I} \Sigma_{(T+I) h v}{ }^{2}-\Sigma_{(T+I) v v} \Sigma_{(T+I) h v}\right) \varepsilon}\right| .
$$

It is evident that $|\Delta \alpha|$ is directly influenced by $\varepsilon$. And the smaller the value of $\varepsilon$ is, the more accurate the angle estimation of the target is.

As is shown in Figure 3, the angle error of RMS (Root Mean Square) caused by the polarization ratio estimation error is drawn via 30 simulation trials by setting the parameters as follows. The polarization ratio of jamming is 3 , the polarization ratio of the target is 5, the DOA of the target is $-0.5^{\circ}$, and the DOA of jamming is $+1.5^{\circ}$. Evidently, the angle estimation error increases with the increasing of the polarization ratio estimation error, while it is not a symmetric relation.

In addition to the polarization ratio estimation error, the target angle estimation accuracy is certainly related to 


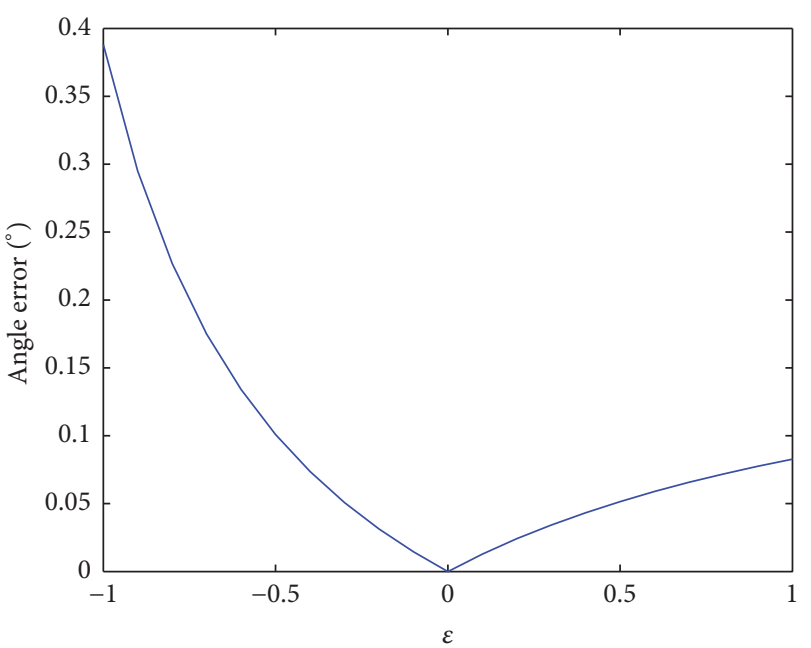

FIGURE 3: Angle estimation error caused by $\varepsilon$.

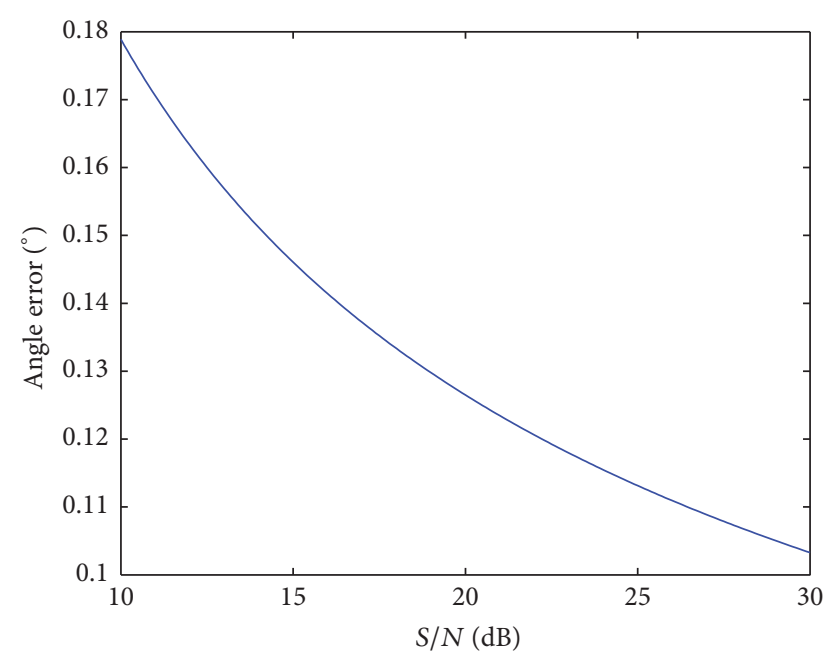

FIGURE 4: Angle error caused by noise.

S/N (SNR, signal-to-noise ratio) of the obtained signal. The relationship can be formulated by

$$
\Delta \alpha_{N}=\frac{\theta_{0.5}}{k \sqrt{B \tau(S / N)\left(f_{r} / \beta_{n}\right)}},
$$

where $\Delta \alpha_{N}$ denotes the angle estimation error related to $S / N, \theta_{0.5}$ denotes the $3 \mathrm{~dB}$ beam width of the radar, $k$ denotes the difference slope constant, $B$ denotes the noise bandwidth of the receiver, $\tau$ denotes the pulse width, $(S / N)$ denotes $S / N, f_{r}$ denotes the pulse repetition frequency (PRF), and $\beta_{n}$ denotes the bandwidth of the radar servo system [21].

On the assumption that $\theta_{0.5}=4^{\circ}, k=0.5, B=$ $100 \mathrm{MHz}, \tau=1 \mu \mathrm{s}, f_{r}=10 \mathrm{kHz}$, and $\beta_{n}=5 \mathrm{kHz}$, the angle error and $S / N$ are related by the curve shown in Figure 4. It is obvious that the angle estimation error decays rapidly with the increasing of $S / N$.

As has been noted above, the angle estimation error is mainly related to the polarization ratio estimation error of jamming and $S / N$ of the obtained signal, whereas $S / N$ is

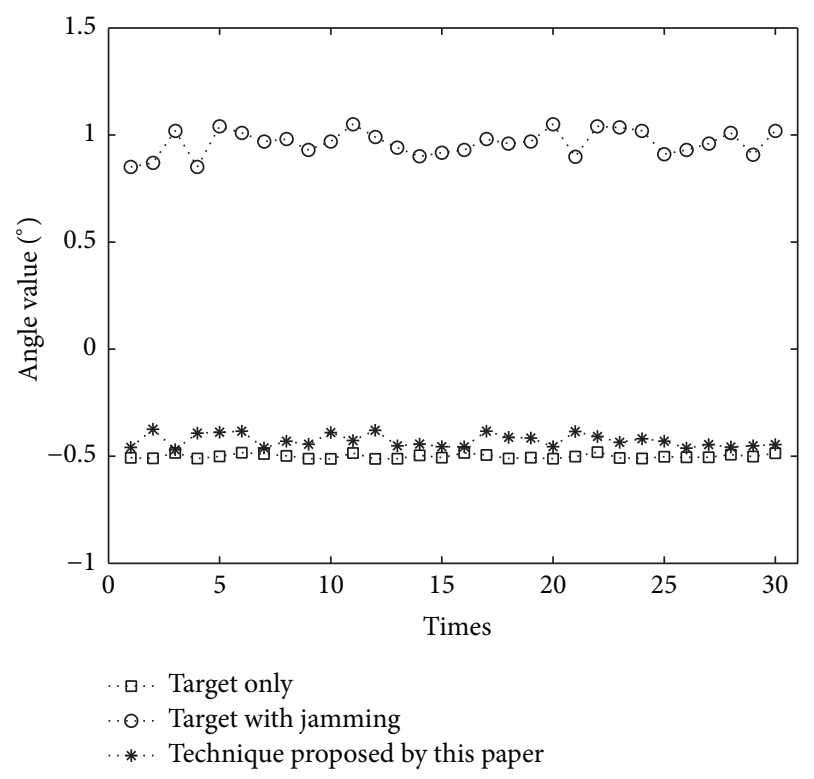

FIGURE 5: Angle estimation of the target being interfered with by the corner reflector.

related to noise factors of the actual physical environment and inherent attributes of the radar system, thus existing objectively. So the key factor to reduce the angle estimation error is the polarization ratio estimation error of jamming. In other words, it is very significant to choose an appropriate estimation technique for the polarization ratio of angle deception jamming.

\section{Experimental Results and Analysis}

In order to prove the effectiveness of the presented technique, two experiments are done with the basic parameters as follows, and all the data analyzed here is experimentally obtained in a real scenario. The dual polarization radar works in the millimeter wave band with the azimuthal beam width of $4^{\circ}$. The target is a small boat, and the corner reflector is approximately three times the RCS of it. Active jamming is of left-hand circular polarization with the transmitting power of $30 \mathrm{dBmW}$, and equivalent RCS of it is approximately five times as large as the boat's. Combined jamming is composed of the corner reflector and the active jamming. The target and the jamming are located as in Figure 1, respectively, at $-0.5^{\circ}$ and $+1.5^{\circ}$ off the boresight of the radar.

The first experiment is done to analyze the feasibility of the presented technique. A comparison of the proposed method with the conventional monopulse method is made. There are, respectively, thirty independent trials for countering the three types of jamming made and shown in Figures 5, 6, and 7, in which, abscissa denotes the sequence number of trials, ordinate denotes the angle estimation value of the target, “ $\square$ " represents the angle estimation value of the target obtained by the conventional monopulse method without angle deception jamming, "o" represents the angle estimation value of the target obtained by the conventional monopulse method with angle deception jamming, and “*” 
TABLE 1: Comparison of the proposed technique with the conventional monopulse method.

\begin{tabular}{|c|c|c|c|c|c|}
\hline & & $\begin{array}{c}\text { Target } \\
\text { only }\end{array}$ & $\begin{array}{l}\text { Target with } \\
\text { corner } \\
\text { reflector }\end{array}$ & $\begin{array}{l}\text { Target with } \\
\text { active } \\
\text { jamming }\end{array}$ & $\begin{array}{c}\text { Target with } \\
\text { combined } \\
\text { jamming }\end{array}$ \\
\hline \multirow{2}{*}{$\begin{array}{l}\text { Conventional } \\
\text { monopulse } \\
\text { method }\end{array}$} & mean & $-0.502^{\circ}$ & $0.967^{\circ}$ & $1.115^{\circ}$ & $1.297^{\circ}$ \\
\hline & $\begin{array}{l}\text { standard } \\
\text { deviation }\end{array}$ & $0.066^{\circ}$ & $0.095^{\circ}$ & $0.098^{\circ}$ & $0.135^{\circ}$ \\
\hline \multirow[b]{2}{*}{$\begin{array}{l}\text { The proposed } \\
\text { technique }\end{array}$} & mean & I & $-0.412^{\circ}$ & $-0.485^{\circ}$ & $-0.458^{\circ}$ \\
\hline & $\begin{array}{l}\text { standard } \\
\text { deviation }\end{array}$ & I & $0.094^{\circ}$ & $0.068^{\circ}$ & $0.090^{\circ}$ \\
\hline
\end{tabular}

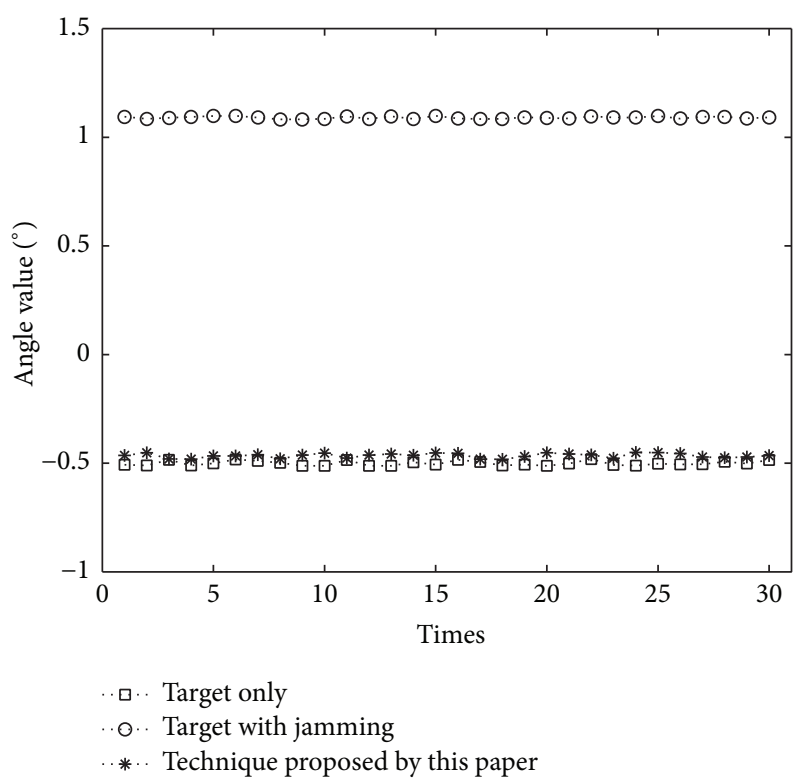

FIGURE 6: Angle estimation of the target being interfered with by active jamming.

represents the angle estimation value of the target obtained by the proposed method with angle deception jamming. And statistical results of the above trials are accorded in Table 1. Making the angle estimation value acquired by the conventional monopulse method without jamming as the standard value of the real target angle, it is obvious that the proposed technique can calculate the actual angle of the target under angle deception jamming with the error in an acceptable range. In particular, the presented technique has a great advantage over the conventional monopulse method.

The second experiment is done to analyze the success rate of tracking with countering the three types of angle deception jamming. Experimental results are shown in Table 2. If the radar does not track to jamming within one minute, it is regarded as success for countering angle deception. Through thirty independent trials of every type of jamming, the proposed technique can achieve success rates of 70\%, $93.3 \%$, and $83.3 \%$, respectively.

Something to note is that the polarization ratio is too important a characteristic for the proposed technique to counter angle deception jamming. For typical radar targets,

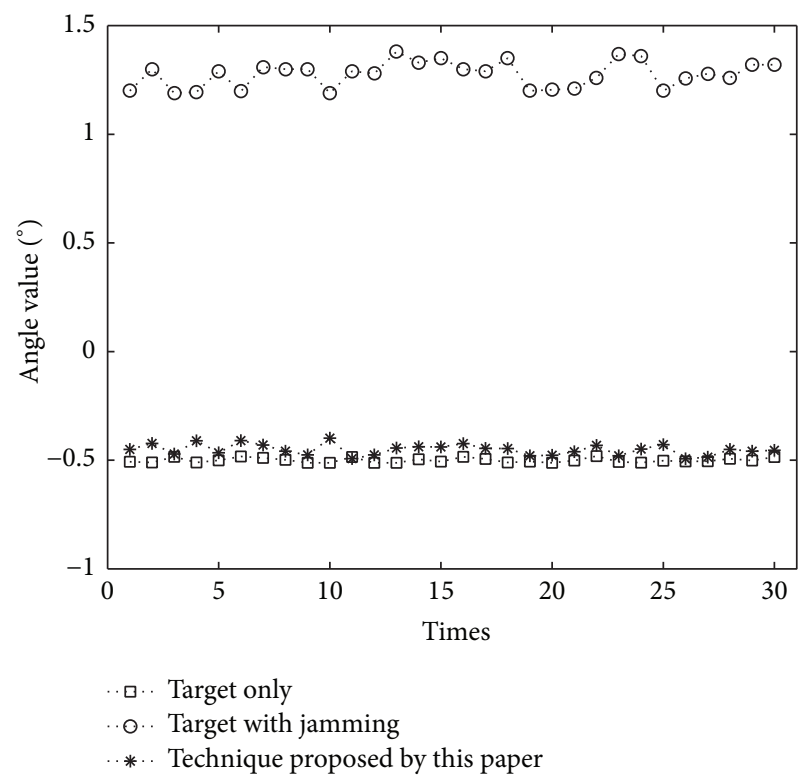

FIGURE 7: Angle estimation of the target being interfered with by the corner reflector and active jamming simultaneously.

TABLE 2: Success rate of countering angle deception jamming.

\begin{tabular}{lccc}
\hline & $\begin{array}{c}\text { Corner } \\
\text { reflector }\end{array}$ & $\begin{array}{c}\text { Active } \\
\text { jamming }\end{array}$ & $\begin{array}{c}\text { Combined } \\
\text { jamming }\end{array}$ \\
\hline $\begin{array}{l}\text { the proposed } \\
\text { technique }\end{array}$ & $70 \%$ & $93.3 \%$ & $83.3 \%$ \\
\hline
\end{tabular}

such as warships and aircrafts, it usually varies from 4 to 8; that of corner reflectors ranges from 7 to 9; that of active jamming with circular polarization approximates 1 ; that of active jamming with vertical polarization is more than 20; however, that of active jamming with horizontal polarization is less than $1 / 20$. Obviously, the polarization characteristic of corner reflectors is close to typical radar targets, while that of active jamming is quite different from them. Therefore, countering active jamming is easier than corner reflectors, which is proved by experimental data in Tables 1 and 2. Though the trials of chaff clouds are not made because of the difficulty of coordination and operation, the proposed technique is also effective for chaff clouds since the polarization ratio of them typically ranges from 3 to 4 [22]. 


\section{Conclusion}

In this paper, an adaptive technique based on dual polarization radar seeker is developed to counter angle deception jamming. The technique is feasible in theory because of its derivation from the conventional monopulse method. Significantly, it does not rely on any prior knowledge of jamming, such as polarization parameters, types, RCS, combined relationship, and so forth. The experimental results certainly prove that the real angle value of the target can be obtained even if it is being interfered with by angle deception jamming, with the angle estimation error in an acceptable range. Polarization technology has a perfect performance for radar seekers to improve target recognition and tracking. Hence its applications will be an interesting investigation in our future work.

\section{Appendix}

\section{Derivation of (12)}

Equations (8) and (10) can be also written as

$$
\begin{aligned}
& k \alpha \Sigma_{T v v}=\Delta_{(T+I) v v}-k \beta \Sigma_{I v v}, \\
& k \alpha \Sigma_{T h v}=\Delta_{(T+I) h v}-k \beta \Sigma_{I h v} .
\end{aligned}
$$

Using (A.1)* $p_{I}$ produces

$$
\begin{aligned}
k \alpha\left(\Sigma_{T v v}-p_{I} \Sigma_{T h v}\right)= & \left(\Delta_{(T+I) v v}-p_{I} \Delta_{(T+I) h v}\right) \\
& -k \beta\left(\Sigma_{I v v}-p_{I} \Sigma_{I h v}\right) .
\end{aligned}
$$

Equations (7) and (9) can be also written as

$$
\begin{aligned}
& \Sigma_{T v v}=\Sigma_{(T+I) v v}-\Sigma_{I v v}, \\
& \Sigma_{T h v}=\Sigma_{(T+I) h v}-\Sigma_{I h v} .
\end{aligned}
$$

Substituting (A.3) into (A.2) gives

$$
\begin{aligned}
k \alpha & \left(\Sigma_{(T+I) v v}-\Sigma_{I v v}-p_{I} \Sigma_{(T+I) h v}+p_{I} \Sigma_{I h v}\right] \\
& =\left(\Delta_{(T+I) v v}-p_{I} \Delta_{(T+I) h v}\right)-k \beta\left(\Sigma_{I v v}-p_{I} \Sigma_{I h v}\right) .
\end{aligned}
$$

From (11), $\Sigma_{I v v}-p_{I} \Sigma_{I h v}=0$, so (A.4) can be simplified as

$$
k \alpha\left(\Sigma_{(T+I) v v}-p_{I} \Sigma_{(T+I) h v}\right)=\left(\Delta_{(T+I) v v}-p_{I} \Delta_{(T+I) h v}\right),
$$

and, then, (12).

\section{Conflicts of Interest}

The authors declare that they have no conflicts of interest.

\section{Acknowledgments}

This work is supported by the scientific research fund of Civil Aviation Flight University of China (Grant no. BJ2016-09) and the National Natural Science Foundation of China (Grant no. U1433126).

\section{References}

[1] W. D. Blair and M. Brandt-Pearce, "Monopulse DOA estimation of two unresolved Rayleigh targets," IEEE Transactions on Aerospace and Electronic Systems, vol. 37, no. 2, pp. 452-469, 2001.

[2] W. D. Blair, G. A. Watson, and M. Brandt-Pearce, "Monopulse processing for DOA estimation of two unresolved Rayleigh targets with known relative RCS," in Proceedings of the 29th Southeastern Symposium on System Theory, SSST, pp. 435-439, IEEE, March 1997.

[3] W. D. Blair and M. Brandt-Pearce, "Unresolved rayleigh target detection using monopulse measurements," IEEE Transactions on Aerospace and Electronic Systems, vol. 34, no. 2, pp. 543-552, 1998.

[4] A. Sinha, T. Kirubarajan, and Y. Bar-Shalom, "Maximum likelihood angle extractor for two closely spaced targets," IEEE Transactions on Aerospace and Electronic Systems, vol. 38, no. 1, pp. 183-203, 2002.

[5] H. Dai, X. Wang, Y. Li, Y. Liu, and S. Xiao, "Main-lobe jamming suppression method of using spatial polarization characteristics of antennas," IEEE Transactions on Aerospace and Electronic Systems, vol. 48, no. 3, pp. 2167-2179, 2012.

[6] H. Dai, X. Wang, J. Luo, Y. Li, and S. Xiao, "A new polarimetric method by using spatial polarization characteristics of scanning antenna," IEEE Transactions on Antennas and Propagation, vol. 60, no. 3, pp. 1653-1656, 2012.

[7] H. Y. Dai, X. S. Wang, Y. Z. Li, J. Luo, and S. P. Xiao, "Spatial polarization characteristics and scattering matrix measurement of orthogonal polarization binary array radar," Science China Information Sciences, vol. 53, no. 12, pp. 2687-2695, 2010.

[8] Y. Yang, D.-J. Feng, W.-M. Zhang, X.-S. Wang, and S.-P. Xiao, "Detection of chaff centroid jamming aided by GPS/INS," IET Radar, Sonar and Navigation, vol. 7, no. 2, pp. 130-142, 2013.

[9] M. Hurtado, J.-J. Xiao, and A. Nehorai, "Target estimation, detection, and tracking: a look at adaptive polarimetric design," IEEE Signal Processing Magazine, vol. 26, no. 1, pp. 42-52, 2009.

[10] MBDA Brimstone missile, http://brimstonemissile.com/ brimstone/.

[11] G. Beard, Performance factors for airborne short-dwell squinted radar sensor [Ph.D thesis], University of London, 2010.

[12] L. Song, H. Qi, X. Qiao, and X. Meng, "Scheme of adaptive polarization filtering based on Kalman model," Journal of Systems Engineering and Electronics, vol. 17, no. 1, pp. 13-18, 2006.

[13] X.-P. Mao, A.-J. Liu, and H.-J. Hou, "Oblique projection polarisation filtering for interference suppression in high-frequency surface wave radar," IET Radar, Sonar and Navigation, vol. 6, no. 2, pp. 71-80, 2012.

[14] R. Boyer, "Oblique projection for source estimation in a competitive environment: algorithm and statistical analysis," Signal Processing, vol. 89, no. 12, pp. 2547-2554, 2009.

[15] Y. Yang, S.-P. Xiao, D.-J. Feng, and X.-S. Wang, "Polarisation oblique projection for radar seeker tracking in chaff centroid jamming environment without prior knowledge," IET Radar, Sonar and Navigation, vol. 8, no. 9, pp. 1195-1202, 2014.

[16] G. Bouleux, "Prior knowledge optimum understanding by means of oblique projectors and their first order derivatives," IEEE Signal Processing Letters, vol. 20, no. 3, pp. 205-208, 2013.

[17] D. K. Barton, Monopulse Radar, Artech House Publisher, Norwood, Mass, USA, 1974. 
[18] M. Skolnik, Radar Handbook, McGraw-Hill Professional, New York, NY, USA, 2008.

[19] J.-S. Lee and E. Pottier, Polarimetric Radar Imaging: From Basics to Applications, CRC Press Inc, Boca Raton, Fla, USA, 1st edition, 2008.

[20] H. Dai, X. Wang, and Y. Li, "Novel discrimination method of digital deceptive jamming in mono-pulse radar," Journal of Systems Engineering and Electronics, vol. 22, no. 6, pp. 910-916, 2011.

[21] D. K. Barton, Modern Radar System Analysis, Artech House Publisher, Norwood, Mass, USA, 1988.

[22] Z. Yinan, J. Ming, Q. Xiaolin, and Z. Zhiquan, "Study on scattering charateristics of chafffor for radar," in Proceedings of the 4th International Conference on Microwave and Millimeter Wave Technology (ICMMT'04), 2004. 


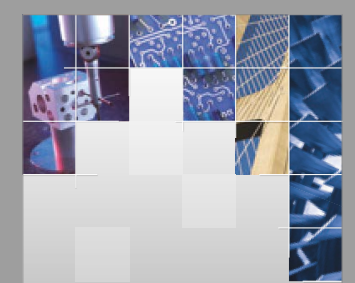

\section{Enfincering}
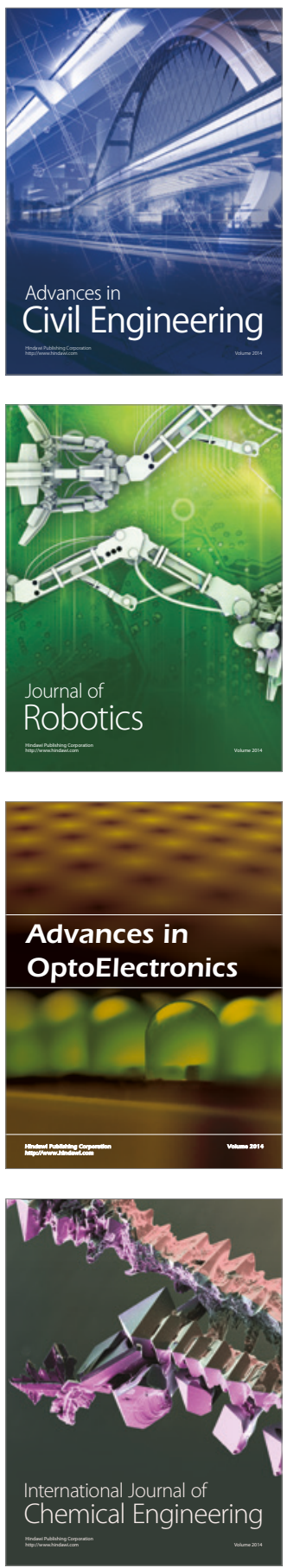

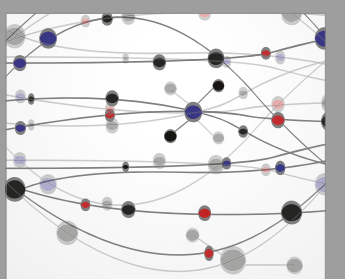

The Scientific World Journal

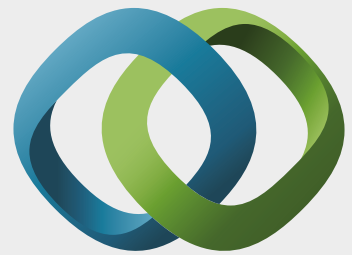

\section{Hindawi}

Submit your manuscripts at

https://www.hindawi.com
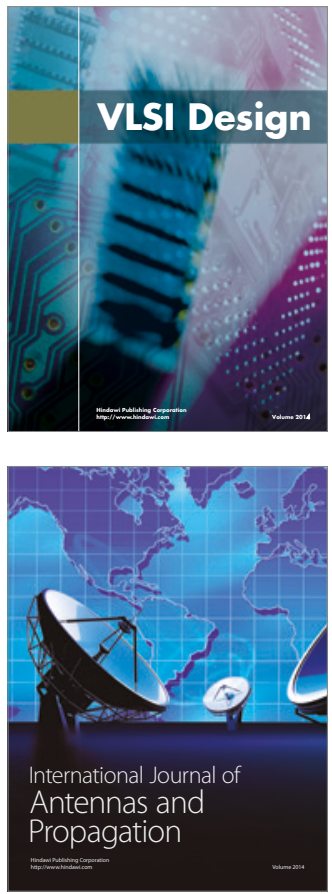

\section{Rotating}

Machinery
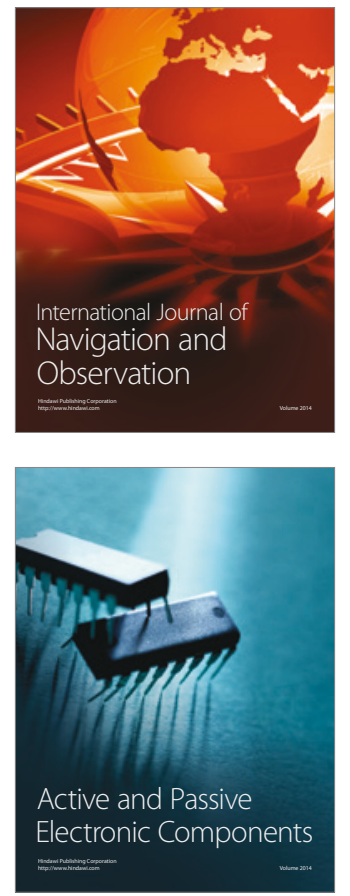
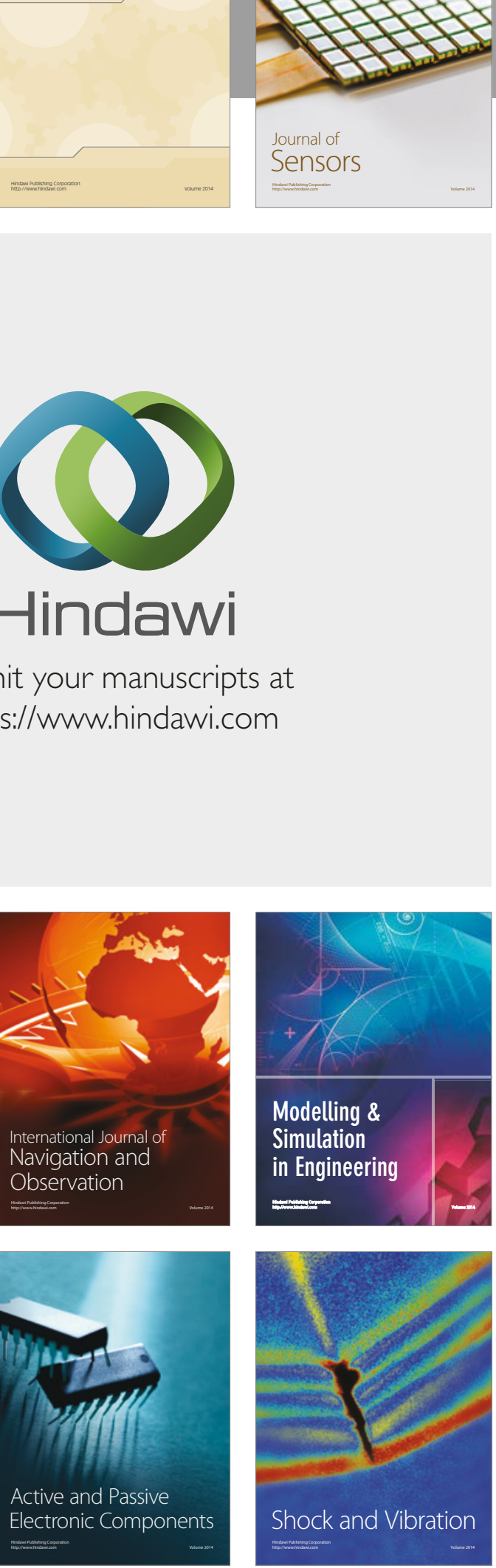
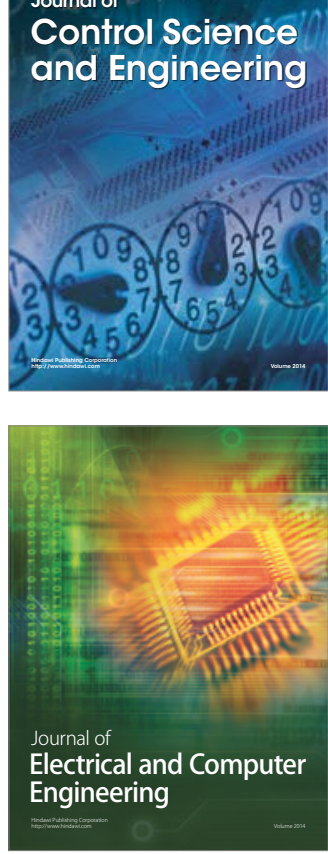

Distributed

Journal of

Control Science

and Engineering
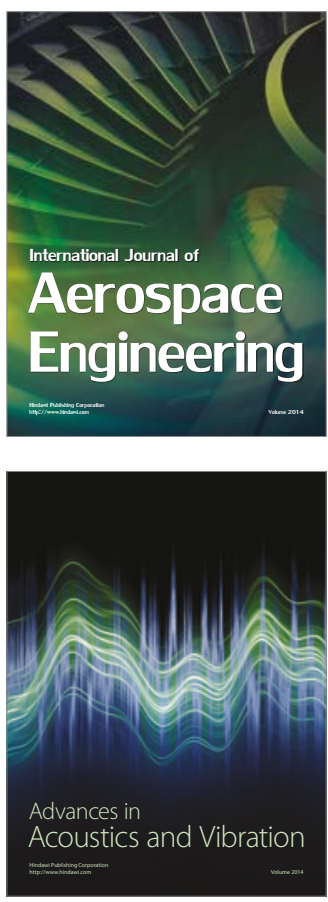

Sensor Networks 